\title{
To share or not to share? Phytoplankton species coexistence puzzle in a competition model incorporating multiple resource-limitation and synthesizing unit concepts
}

\author{
Subrata Sarker ${ }^{\mathrm{a}, *, 1}$, Ulrike Feudel ${ }^{\mathrm{b}}$, Cédric L. Meunier ${ }^{\mathrm{a}}$, Peter Lemke ${ }^{\mathrm{c}}$, Partha S. Dutta ${ }^{\mathrm{d}}$, \\ Karen H. Wiltshire ${ }^{\text {a }}$ \\ a Alfred-Wegener-Institut Helmholtz-Zentrum für Polar- und Meeresforschung, Biologische Anstalt Helgoland, Helgoland, Germany \\ b Theoretical Physics/Complex Systems, ICBM, Carl von Ossietzky Universität, PF 2503, 26111, Oldenburg, Germany \\ ${ }^{c}$ Alfred-Wegener-Institut Helmholtz-Zentrum für Polar- und Meeresforschung, Bremerhaven, Germany \\ ${ }^{\mathrm{d}}$ Department of Mathematics, Indian Institute of Technology Ropar, Rupnagar, Punjab, India
}

\section{A R T I C L E I N F O}

\section{Keywords:}

Coexistence

Co-limitation

Nutrients

Model

Helgoland Roads

\begin{abstract}
A B S T R A C T
The predictions of the competitive exclusion principle about the number of coexisting species not exceeding the number of limiting resources in equilibrium constitute an ecological puzzle for phytoplankton ecosystems. Here we present a synthesizing unit (SU) based competition model taking co-limitation into account, which is the extension of the competition model developed by Dutta et al. (2014).The study aims at understanding the mechanisms of violation of competitive exclusion principle for phytoplankton species with seasonal environmental forcing when multiple resource limitation is taken into account and species growth is formulated based on SU. We also explore the role of changing environmental conditions on species coexistence on a seasonal and a decadal time scale by linking the model forcing to the Helgoland Roads Time Series data sets. For the first time, based on the Helgoland Roads data, we are able to find a realistic parameterization for the phytoplankton competition model where growth is formulated using SU concept. Our study confirms that more species than limiting resources can coexist with seasonal variations of environmental conditions. This supersaturation is related to periodic changes in species' biomass, variation in interspecific competition and niche configuration, nonlinear functional response and the position of resource supply within the convex hull of species' resource uptake rate. Changes in environmental conditions within realistic ranges do not prevent the coexistence of species rather it slightly changes species' biomass and turnover time. This study also confirms that our model with SU based species growth performs better than species competition model where multiple resource limitation is formulated based on the product of several Monod functions. Our study has created a new avenue for phytoplankton coexistence research and the results might be helpful to answer the complex questions on species diversity maintenance in nature.
\end{abstract}

\section{Introduction}

Understanding species coexistence is one of the most important fundamental research objectives in community ecology (Hartig et al., 2014; Laird and Schamp, 2006). The mechanisms of species coexistence have long puzzled ecologists (Segura et al., 2011) and the greatest challenge lies in reconciling of coexistence with the competitive exclusion principle (Laird and Schamp, 2006). The competitive exclusion principle (Hardin, 1960) states that the maximum number of coexisting species cannot exceed the number of limiting resources in equilibrium.
This claim however contradicts the observations of species number in plankton communities, which led to the formulation of the paradox of plankton (Hutchinson, 1961). Hutchinson (1961) pointed out that in planktonic systems many phytoplankton species can coexist while it seemed that only a few resources (i.e. light and nutrients) are limiting. Since the formulation of the paradox of plankton, many theories (i.e. niche theory, neutral theory and lumpy coexistence) have been proposed to explain species coexistence. Niche theory suggests that multiple species can coexist if the resources they require for growth differ sufficiently (Vandermeer, 1972). However, neutral theory of species

\footnotetext{
* Corresponding author at: Alfred-Wegener-Institut Helmholtz-Zentrum für Polar- und Meeresforschung, Biologische Anstalt Helgoland, 27498, Helgoland, Germany.

E-mail addresses: subrata.sarker@awi.de, subratasrk-ocg@sust.edu (S. Sarker).

${ }^{1}$ Current affiliation: Department of Oceanography, Shahjalal University of Science and Technology, Sylhet-3114, Bangladesh.
} 
coexistence, which deviates from niche theory, suggests that high diversity can be maintained even when life-history traits are very similar (Hubbell, 2001). A reconciliation of niche and neutral theory, termed "lumpy coexistence" (Sakavara et al., 2017), describes the self-organization of assemblages into competing clumps, in which species within clumps have very similar traits and are thus considered nearly neutral (Scheffer and van Nes, 2006). For the number of species to exceed that of resources, termed "supersaturated coexistence" (Schippers et al., 2001), species should differ in their resource-use abilities (Dutta et al., 2014). Since the formulation of the competitive exclusion principle a large number of modelling studies have been conducted to explain the species coexistence taking resource limitation into account.

There are two basic concepts to modelling resource limited species dynamics in the presence of several resources: Liebig's law of the minimum (Liebig, 1840) and the multiple resource limitation hypothesis (Gleeson and Tilman, 1992). Liebig's law of the minimum (Liebig, 1840) states that only a single resource limits species' growth at any given time. However, this idea has been replaced by the realization that phytoplankton growth can be limited by several resources simultaneously. Supersaturated coexistence of species in a multiple resources limited environment based on Liebig's law of the minimum and the product of several Monod functions both lack a good fit to data (Poggiale et al., 2010; Sperfeld et al., 2012). Thus, Dutta et al. (2014) developed a new approach allowing "supersaturated coexistence" of species in a multiple resource-limited ecosystem, based on the concept of a synthesizing unit (SU). This SU converts resources into biomass, fulfilling all stoichiometric requirements for the biomass formation (Kooijman, 2010). A SU forms a product according to the rules prescribed by classical enzyme kinetics, with some modifications: the kinetics is specified in terms of arrival fluxes of the substrate molecules to the enzyme, not in terms of substrate complexes. The enzyme-substrate dissociation rate is assumed to be zero and a SU can bind an arbitrarily large number of substrates and transfer them into products. Moreover, recent experimental results on resource co-limitation theory applied to herbivorous consumers have shown that species growth kinetics based on the concept of SU fits the data better than the product law growth rate (Sperfeld et al., 2012). In addition, this approach obeys mass of conservation. All the nutrient uptake processes are assumed to be irreversible. Such models produce all known possible outcomes of competition (i.e. competitive exclusion, heteroclinic cycles, and equilibrium). As Schippers et al. (2001) pointed out the model of Huisman and Weissing (1999) lacks robustness and supersaturation collapses when parameter are changed only slightly. It is important to note that the re-formulation of this model using the SU-unit overcomes this drawback and exhibits supersaturation in large parameter intervals as demonstrated in Dutta et al. (2014).

Due to the prediction of competitive exclusion principle species coexistence in a limited number of resources has been studied widely (e.g. Roelke et al. (2003), Roelke and Eldridge (2008), Roelke and Eldridge (2008)). However, studies on phytoplankton species coexistence in a multiple resources limited ecosystem based on the concept of a synthesizing unit are rare. The parameterization of the model developed by Dutta et al. (2014) is based on a bacteria-nutrients system and is therefore not suitable to study the competition of phytoplankton species. To test "supersaturation" to be a real possibility for the coexistence of phytoplankton species needs a completely new parameterization of that model, including the check that this new parametrization is robust. Moreover, no previous modelling studies on species coexistence compared the output of species competition with real data sets. Many studies found different behavior in species dynamics in the supersaturated state (e.g. identity of the dominant species changes, cycles in species abundance with peaks every couple of years etc.). These findings might hold true for a real data set. For example, long-term phytoplankton data sets from the Helgoland Roads Time Series also show (Fig. 1) that different species might behave differently on the long-term (i.e. a few species peak in species abundance after few years interval and a few species peak every year). Therefore, comparison of model outputs with the observed data might offer more realistic and exciting insights on species coexistence from the theoretical studies. Here, for the first time, we test the possibility whether the number of coexisting phytoplankton species can exceed the number of known limiting resources in a shallow-sea ecosystem taking the simultaneous co-limitation based on the concept of a SU into account. We also test the behaviour of long-term species coexistence by changing environmental forcing based on the Helgoland Roads Time Series data sets (Raabe and Wiltshire, 2009; Wiltshire and Dürselen, 2004). In addition, we discuss our species dynamics produced by the model with the real phytoplankton data (Fig. 1) from the Helgoland Roads Time Series Station.

This study aims to answer the following questions:

(i) Does the number of coexisting phytoplankton species, exceed the number of limiting resources in a multiple resource limited ecosystem modelled by formulating species growth using a SU based approach?

(ii) If yes, then what causes this supersaturated coexistence in an ecosystem where species growth is formulated using a SU?

(iii) What are the consequences of long-term coexistence of species?

(iv) What happens to species coexistence if environmental forcing is changed?

(v) Does the species competition model with SU approach performs better than the model with Monod equations?

\section{Materials and methods}

\subsection{Model description}

The main goal of our study is to explain the phytoplankton species coexistence in a multiple resource limited ecosystem by formulating species growth based on the SU concept. More specifically, we aim at understanding how the number of phytoplankton species coexisting can exceed the number of limiting resources. To do so, we based our work on the species competition model developed by Dutta et al. (2014). Dutta et al. (2014) presented a multiple species-multiple resource competition model which is based on the concept of synthesizing unit to formulate the growth rates of species competing for interactive essential resources. They demonstrated that a more mechanistic explanation of interactive effects of co-limitation may lead to the known complex dynamics including non-equilibrium states as oscillations and chaos. This competition model described a community in a chemostat and seasonal forcing was not used. Moreover, no temperature and light dependence of the growth rate of the species was introduced being a necessary condition for using this model to study the competition of phytoplankton species. Therefore, we extended this model for phytoplankton species in a shallow-sea ecosystem. A schematic representation of the model with the flows between variables and parameters is shown in Fig. 2. So, how does our model differ from Dutta et al. (2014)? We considered a shallow sea ecosystem while Dutta et al. (2014) considered the chemostat system where bacteria compete for nutrients. We consider phytoplankton as model organisms and identify a new parameter set which is based on observed data. In addition, seasonal temperature and light forcing is introduced in our case which were absent in Dutta et al. (2014). Species growth rates are formulated as a function of nutrients, temperature and light in our model. We use four phytoplankton species, and $\mathrm{SiO}_{2}, \mathrm{PO}_{4}$ and $\mathrm{NO}_{3}$ as three resources in the model. We take their initial conditions from the Helgoland Roads Time Series data sets.

The model considers interactive effects of three essential nutrients $\left(\mathrm{SiO}_{2}, \mathrm{PO}_{4}\right.$ and $\left.\mathrm{NO}_{3}\right)$, temperature and light on the phytoplankton species dynamics. The model equations for $n$ species and $k$ nutrients in a shallow-sea system are described as: 


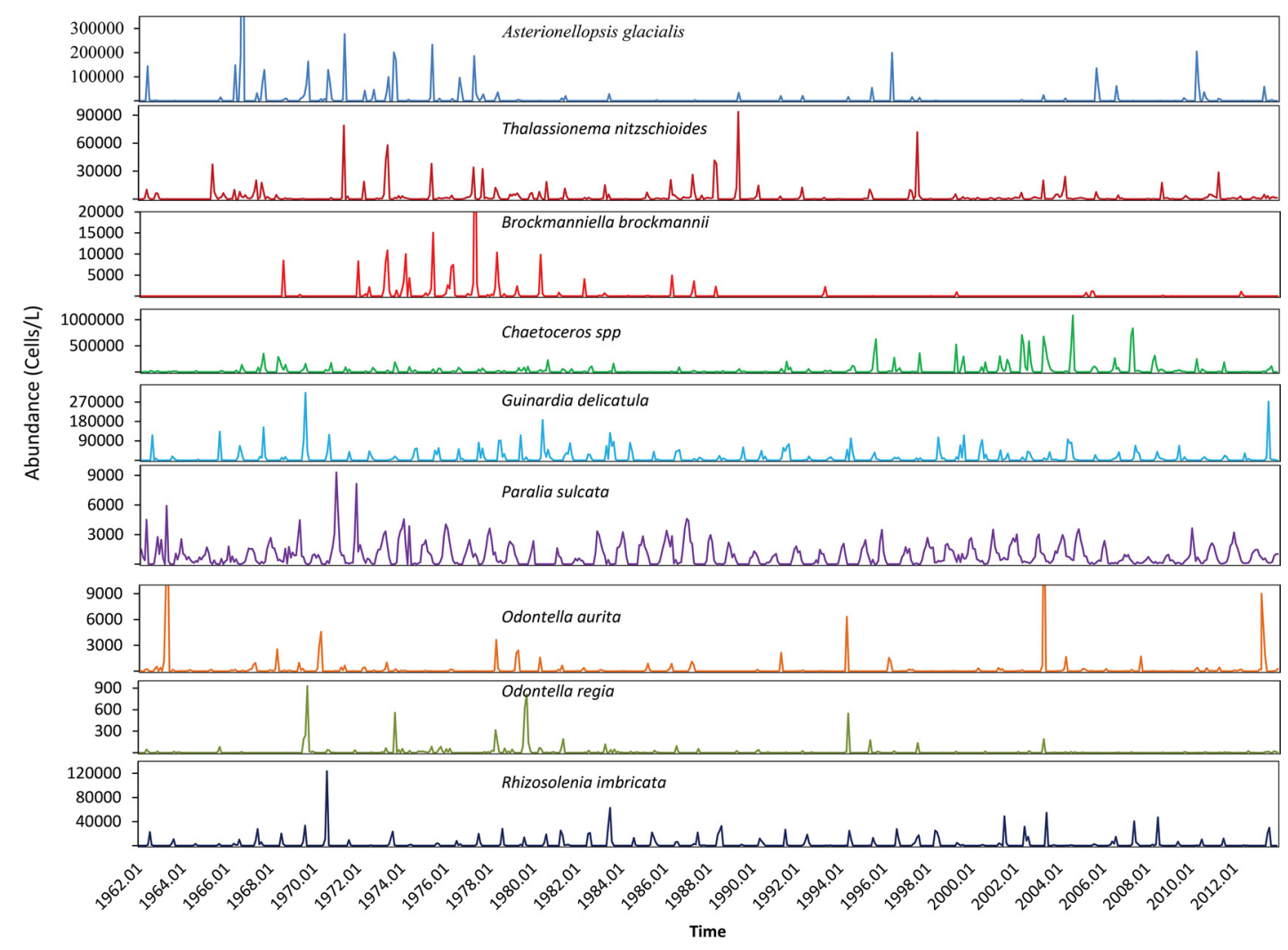

Fig. 1. Long-term monthly abundance of some selected phytoplankton species from the Helgoland Roads Time Series data sets (Wiltshire and Dürselen, 2004).

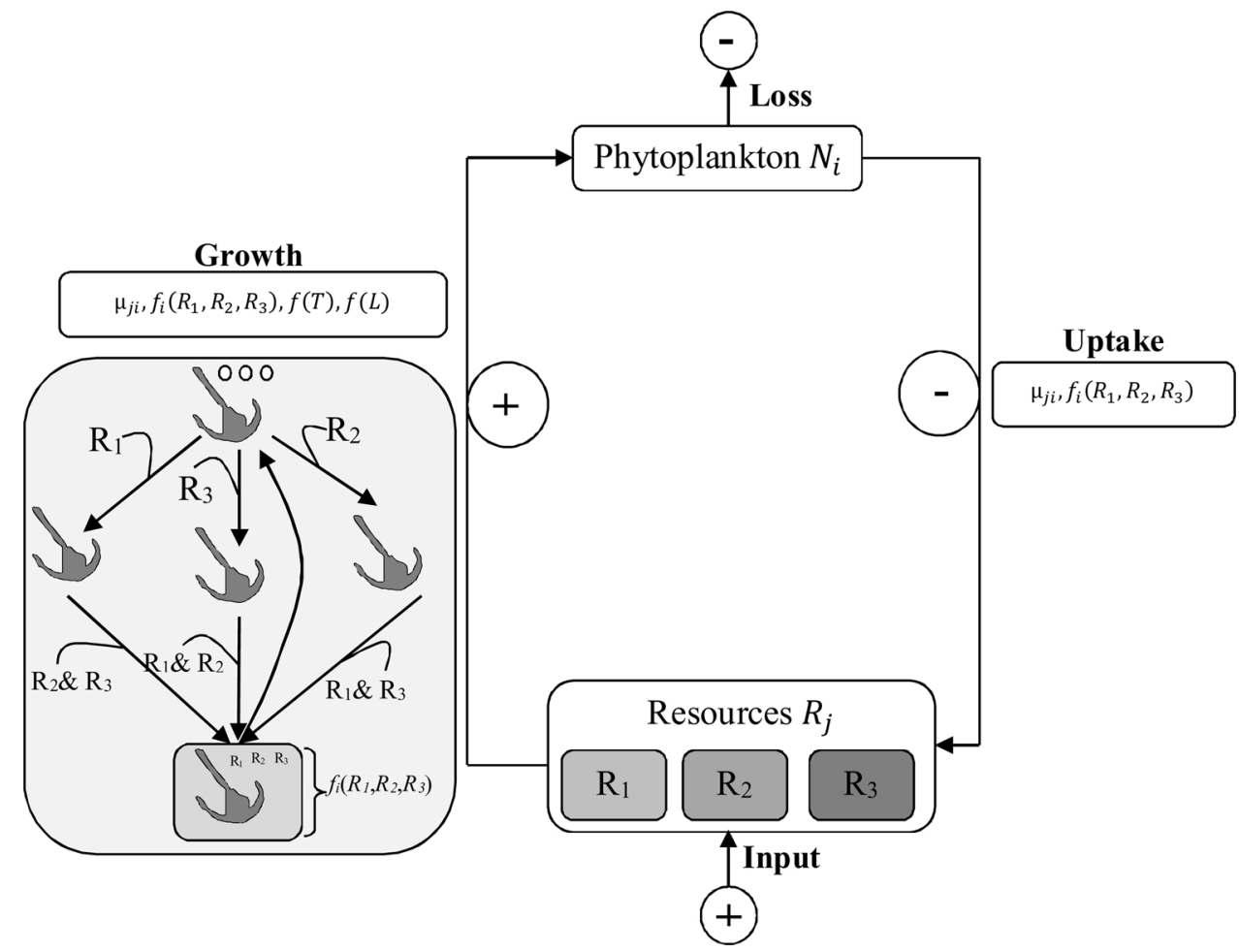

Fig. 2. Conceptual diagram of the multiple-resource competition model for phytoplankton. Phytoplankton growth depends on the maximum growth for a specific resource $\mu_{j i}$, functional response to the resources $f_{i}\left(R_{1}, R_{2}, R_{3}\right)$, temperature $f(T)$ and light condition $f(L)$. Phytoplankton growth based on resource availability is formulated based on a Synthesizing Unit (SU) approach and illustrated here inside of the shaded box (for details see the model description in the method section of the manuscript). 
$\frac{d N_{i}}{d t}=N_{i}\left(\sum_{j=1}^{k} G_{j i}-\left(k+m_{i}\right)\right) i=1,2, \ldots, n$

$\frac{d R_{j}}{d t}=k\left(S_{j}-R_{j}\right)-\sum_{i=1}^{n} I_{j i} N_{i} j=1,2, \ldots, k$

Where $N_{i}$ denotes the biomass of species $i$ and $R_{j}$ denotes the concentration of resource $j$. The dynamics of species $i,\left(\frac{d N_{i}}{d t}\right)$, includes growth $G_{j i}$, mortality $m_{i}$, and the dynamics of resource $j,\left(\frac{d R_{j}}{d t}\right)$, includes the nutrient input from a reservoir below the mixed layer $S_{j}$ with a thermocline exchange rate $K$ and the uptake rate of resource $j, I_{j i}$ by phytoplankton species $i$.

The key part of the model is the formulation of the phytoplankton species' growth $G$ Eq. (3) as a function of nutrients, temperature and light based on a SU approach, as well as, the nutrients uptake rate Eq. (4).

$G_{j i}=\mu_{j i} f_{i}\left(R_{1}, R_{2}, R_{3}\right) f(T) f(L)$

$I_{j i}=U_{j i} f_{i}\left(R_{1}, R_{2}, R_{3}\right)$

where $\mu_{j i}$ is the maximum growth rate of species $i$ for resource $j$ and $U_{j i}$ is the maximum uptake rate of nutrient $j$ by species $i$. The term $f_{i}\left(R_{1}, R_{2}, R_{3}\right)$ is the functional response of species $i$ to $k$ nutrients based on SU (Dutta et al., 2014; Kooi et al., 2004; Muller et al., 2001). The corresponding functional response for species $i$ to three nutrients $\left(\mathrm{SiO}_{2}\right.$, $\mathrm{PO}_{4}$ and $\left.\mathrm{NO}_{3}\right)$ with their half saturation constants $\left(K_{1 i}, K_{2 i}\right.$ and $K_{3 i}$ respectively) and the concentration of the 3 resources in the water column ( $R_{1}, R_{2}$ and $R_{3}$ respectively) can be written as follows according to Dutta et al. (2014).

$$
f_{i}\left(R_{1}, R_{2}, R_{3}\right)=\frac{1}{1+\frac{K_{1 i}}{R_{1}}+\frac{K_{2 i}}{R_{2}}+\frac{K_{3 i}}{R_{3}}-\frac{1}{\frac{R_{1}}{K_{1 i}}+\frac{R_{2}}{K_{2 i}}}-\frac{1}{\frac{R_{2}}{K_{2 i}}+\frac{R_{3}}{K_{3 i}}}-\frac{1}{\frac{R_{1}}{K_{1 i}}+\frac{R_{3}}{K_{3 i}}}+\frac{1}{\frac{R_{1}}{K_{1 i}}+\frac{R_{2}}{K_{2 i}}+\frac{R_{3}}{K_{3 i}}}}
$$

In Eq. (3), $f(T)$ and $f(L)$ are factors expressing the impact of temperature and light on the growth rate of the species. Eq. (5) is associated to complementary resources with sequential binding. Phytoplankton can uptake nutrients from the aquatic medium and can transfer them into biomass. To illustrate this concept in a simple way, we consider here a SU with three substrates or nutrients (i.e. $R_{1}, R_{2}$ and $R_{3}$ ). Depending on which resource arrives first, either $R_{1}$ or $R_{2}$ or $R_{3}$ will be taken up by phytoplankton in the first step. Subsequently, $R_{2}$ or $R_{3}, R_{1}$ or $R_{3}, R_{1}$ or $R_{2}$ will be taken up in the second step and the remaining one in the third step, respectively. Biomass can only be formed when three resources $\left(R_{1}, R_{2}\right.$ and $\left.R_{3}\right)$ are bound to the SU.

The effect of temperature on the species growth rate is described by the van't Hoff rule and thus the temperature function $f(T)$ is written as:

$f(T)=\left(Q_{10}\right)^{(T(t)-10) / 10}$

Eq. (6) asserts that a change of the temperature by $10^{\circ}$ will multiply the rate at mean temperature by a factor $Q_{10}$. For phytoplankton species $Q_{10}$ varies between 1.3 and 4 (Freund et al., 2006). The temperature $T(t)$ is the seasonal temperature forcing and described as follows Eq. (7) according to Freund et al. (2006).

$T(t)=T_{m}+\Delta T \sin (\Omega t+\varphi)$

where mean temperature $T_{m}=10.10{ }^{\circ} \mathrm{C}, \Delta T=6{ }^{\circ} \mathrm{C}, \Omega=2 \pi /(365$ days), and $\varphi / 2 \pi=0.59$ were adapted from a fit to the Helgoland Roads time series data sets.

The light function $f(L)$ expresses the effect of light on species' growth and can be written according to Li et al. (2010):

$f(L)=\left[1-\exp \left(\frac{-\alpha I_{P A R}(t)}{\delta}\right)\right] \exp \left(\frac{-\beta I_{P A R}(t)}{\delta}\right)$

where $\alpha$ and $\beta$ are the initial slope of the P-I curve and the photo inhibition coefficient respectively, and $\delta$ denotes the species' maximum specific growth rate under light saturation. All the simulations were performed using a fixed set of parameters values (Table S1) for the phytoplankton ecosystem. $I_{P A R}$ is calculated as a function of time dependent sunshine hours $(S H)$ and Secchi depth $(S D)$ as follows using the formula from French et al. (1982).

$I_{P A R}(t)=7.5 S H(t) e^{-\frac{1.7}{S D(t)}}$

Both sunshine hours $S H(t)$ and Secchi depth $S D(t)$ are taken as a seasonal forcing described as Eq. (10) and Eq. (11) respectively.

$S H(t)=S H_{m}+\Delta S H \sin \left(\Omega_{S H} t+\varphi_{S H}\right)$

$S D(t)=S D_{m}+\Delta S D \sin \left(\Omega_{S D} t+\varphi_{S D}\right)$

where mean sunshine hours $S H_{m}=5.51, \Delta S H=2.98, \Omega_{S H}=2 \pi /(365$ days), $\varphi_{S H} / 2 \pi=0.75$, mean Secchi depth $S D_{m}=3.79, \Delta S D=1.59$, $\Omega_{S D}=2 \pi /(365$ days $)$, and $\varphi_{S D} / 2 \pi=0.75$ were adapted from a fit to the Helgoland Roads time series data sets.

\subsection{Observed data}

We used the Helgoland Roads Time Series data sets to apply a realistic environmental forcing and to set the initial conditions in the model. We also used this data set also to compare the species' dynamics produced in our competition model. The Helgoland Roads Time Series Station $\left(54^{\circ} 11.3^{\prime} \mathrm{N}, 7^{\circ} 54.0^{\prime} \mathrm{E}\right)$ is one of the long-term ocean monitoring sites of Biologische Anstalt Helgoland which was started in 1962. Work daily monitoring of biological, chemical and physical parameters has been carried out continuously at this station since it has started, and is one of the longest aquatic data sets in history (Wiltshire and Dürselen, 2004). Water samples are collected from the surface, and temperature and Secchi depth (as a measure of transparency) are measured in situ. Samples are preserved for further analysis of nutrients, phytoplankton and zooplankton. The nutrients (phosphate, ammonium, nitrate and nitrite) are measured using the standard colorimetric methods described by Grasshoff and Almgreen (1976) immediately on a filtered sub-sample from the daily Helgoland Roads surface water sample (Wiltshire et al., 2010).

We split the long-term Helgoland Roads data sets from 1962 to 2010 into 5 time blocks i.e. 1962-1970, 1971-1980, 1981-1990, 1991-2000 and 2001-2010. We then calculated the average values of all abiotic and biotic factors for each time block. By taking these averaged values as environmental forcing we run one model for each time block (environmental conditions for each time block is shown in Table S2). The objective of running five models with five different environmental forcings from different time periods of the Helgoland Roads data set was implemented to see how different environmental forcing affects the species dynamics and coexistence in the model.

\subsection{Parameter estimations}

To estimate a range for each parameter value we performed a Markov chain Monte Carlo (MCMC) simulation (Gilks et al., 1995) on observed species data in the OpenBUGS software (Thomas et al., 2006). These species correspond to the dominant functional group diatom in the field database. The MCMC is the most popular approach to evaluating posterior distributions of model parameters. The principle of MCMC is to generate a large sample from a distribution of interest (usually the joint posterior), and base inferences on the simulated sample. For example, the posterior mean and variance of a given quantity can be estimated by the mean and variance of an MCMCgenerated posterior sample. This involves with the specification of priors of the model parameters i.e. species growth rate, resource uptake rate, mortality rate etc. Then MCMC simulations give the posterior distribution of each parameter at 95\% confidence interval based on Gibbs sampling. The posterior distributions of each parameter include a 
range of values within which the real value of that parameter might be located. To do this, the OpenBUGS software was linked with the statistical computing software R (R Development Core Team, 2010) by the R2OpenBUGS package (Sturtz et al., 2005) for the analysis. Later with these posterior distributions of all parameters, we performed a Monte Carlo search to find a parameters set where four species can coexist in three limiting resources. This way of estimation gives more reliable values of model parameters rather than taking parameter values arbitrarily.

\subsection{Model evaluation technique}

To evaluate which model perform better, either the competition model with Liebig's law of minimum or the competition model with SU approach, we performed the following analyses:

Firstly, we observed the dynamics of species growth described by a functional response based on synthesizing unit with multiple resource limitation (Eq. (12)) and a product of several Monod functions with multiple resource limitation (Eq. (13)). This observation will show how the growth of biomass with the SU changes in comparison with the product of several Monod functions. Secondly, we tested whether all known possible outcomes of competition predicted by previous models can be reproduced or not by our phytoplankton species competition model considering SU with multiple resource limitation. Thirdly, we tested how sensitive the models are to changes in parameter values. The better model should be able to produce a supersaturation state even in a large variation in parameter values in comparison to other models.

$f_{c}=\frac{1}{1+\left(\frac{R_{1}}{K_{1}}\right)^{-1}+\left(\frac{R_{2}}{K_{2}}\right)^{-1}-\left(\frac{R_{1}}{K_{1}}+\frac{R_{2}}{K_{2}}\right)^{-1}}$

$f_{c}=\frac{1}{\left(1+\frac{K_{1}}{R_{1}}\right)\left(1+\frac{K_{2}}{R_{2}}\right)}$

where $f_{c}$ is the functional response, $K_{1}$ and $K_{2}$ are the concentrations of $R_{1}$ and $R_{2}$ resources respectively.

\section{Results}

The results of our simulations are summarized under the guise of our overarching questions.

\subsection{Does the number of species coexists exceed the number of limiting resources when growth is modelled based on a SU?}

Our main objective of this paper was to study the possibility of more species coexistence than the number of limiting resources using the competition model based on SU-based growth rate with the multiple resource limitation hypothesis. Our model simulations confirm that more phytoplankton species than resources may coexist while considering multiple resource limitation and species growth is formulated based on SU approach. We emphasize that in contrast to previous studies this coexistence persists when taking a seasonal variation of environmental condition into account.

Fig.3a shows different oscillatory states of four species biomass over time. This suggests that simultaneous co-limitation of multiple resources formulated using the SU concept does not prevent the emergence of a supersaturated coexistence of species.

\subsection{What causes supersaturated coexistence in a multiple resource limited ecosystem?}

We found the following reasons for supersaturated coexistence of species in the model where species growth is formulated based on a SU approach:

Non-equilibrium state: Competition for resources generates periodic oscillations in species biomass and allows the coexistence of more species than the number of resources. Here, for silicate, species 1 has the lowest half saturation constant $(k)$ and species 4 has maximum growth rate $(\mu)$. Therefore, species 1 is a strong competitor for silicate but species 4 is limited by silicate. Similarly, species 2 is a strong competitor for phosphate but species 3 is limited by phosphate. Species 3 is a strong competitor for nitrate but species 1 is limited by nitrate. Species 4 is the intermediate competitor for phosphate and nitrate. This type of competition for three resources by four phytoplankton species (i.e. if a species is a strong competitor for one resource then its' growth is limited by another resource) generates cyclic dynamics in their biomass and leads to a cyclic succession of species.

Resource requirements, competition and niche differentiation: In our model, if one species has maximum requirements for a particular resource, then it has intermediate and minimum requirements for other resources. These types of resource requirements i.e. differences in feeding traits is one of the important reasons for the observed supersaturation.

In addition, differences in resource requirements of species indicate the variation in their interspecific competition ability. This suggests that the four species considered in the model have different niche configuration and thus, competitive exclusion does not occur.

Functional response of species: In our model species exhibit nonlinear functional response. This functional response causes species growth rates to relate to resource abundance in a nonlinear manner. As resources fluctuate over time the species with the more nonlinear functional response is better at exploiting the resource whose abundance is lower and the species with the less nonlinear functional response is better for exploiting the resource when resource abundance is higher. Thus, temporal fluctuations in the resource allow coexistence via resource partitioning.

Nutrient uptake rate and supply: In our model the nutrient supply $S_{j}$ lies within the convex hull of resource uptake by species. Thus, competitive exclusion does not apply and instead, the species "cooperate" in creating an environment equally favorable for all.

\subsection{What are the consequences of long-term supersaturated coexistence of species?}

To understand the consequences of long-term supersaturated coexistence, we closely observed the species dynamics generated by our model. The model indicates that there is always one dominant species while the other three species have lower biomasses. Therefore, they do coexist. Further, the identity of the dominant species changes over time within the community. The seasonal forcing applied in the model results in a biomass fluctuation periodicity which repeats every one or some years later (Fig. 4). Species with the periodicity of $P$ number of years produce $P$ lines in the phase plane diagram before it returns to its starting point. In the phase plane diagrams (Fig. 4), continuous trajectories of species biomass indicate the characteristic oscillation within the community. This continuous oscillation in each species biomass yields an oscillation of the total biomass with nearly constant amplitude (Fig. 3C). The periodicity in species succession within the community in multiple resource limited ecosystem supports the possibility of supersaturated coexistence.

\subsection{What happens to species coexistence if environmental forcing is changed?}

In order to understand how changes in environmental conditions (i.e. nutrients, temperature and light) affect species coexistence, we changed the model forcing based on the Helgoland Roads Time Series data sets (i.e. environmental forcing of 1970 s, 1980 s, 1990 s, 2000 s and $2010 \mathrm{~s}$ ). Our simulations show that, despite changes in environmental forcing, all four species can coexist when competing for three resources (Fig. S1). In addition, these changes have also no significant 

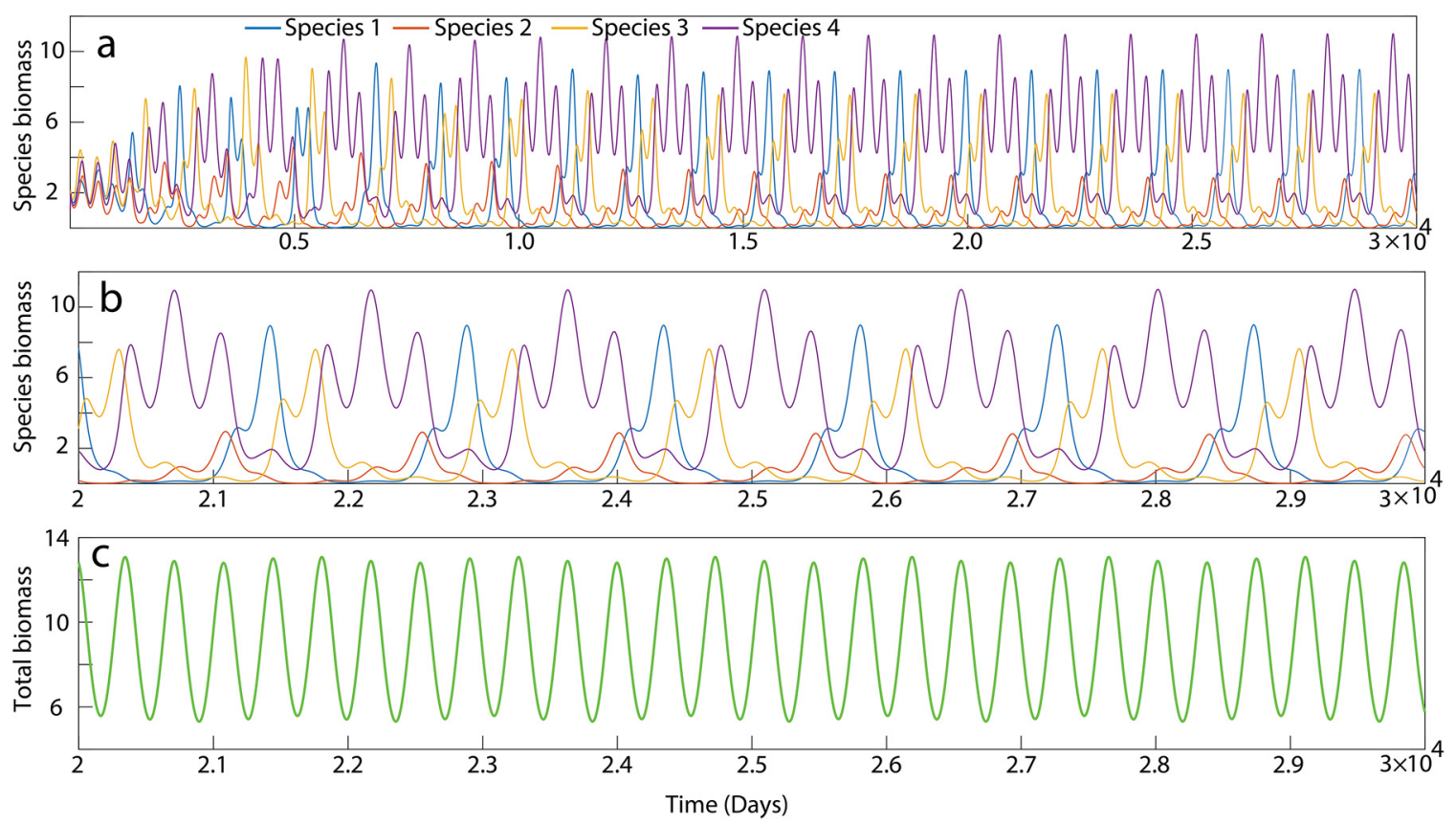

Fig. 3. Coexistence dynamics of four species competing for three resources. (a) Long-term behavior of four species biomass. (b) Four species dynamics during the last 10,000 days of the simulation. (c) The corresponding total biomass of four species during the last 10,000 days.

impact on species biomass dynamics i.e. only a little variation in species dynamics was observed. With different environmental forcing, species produce different types of limit cycles in phase plane diagram (Fig. 5). The species succession pattern in different simulations remains similar but the dominant species identity changes within the same forcing over time (Fig. 6). We have also shown that in a supersaturated state the turnover time of a species might vary. For example, if one species shows a peak at the beginning of the year, that species might not show the peak at the same time of the next year (Fig. 7). In addition, when a species enters in its long-term behavior, it might show a major peak after some year's interval, for example one major peak in every fourth year (Fig. 7).

\subsection{Does the species competition model with SU approach performs better than the model with Monod equation?}

To identify whether phytoplankton species competition model described by SU with multiple resource limitation performs better than the model described by the product of several Monod functions with multiple resources limitation, we performed three tests as described in Section 2.4. Firstly, we found that the use of the product of several Monod functions with multiple resources limitation has a methodological disadvantage. The use of the product of several Monod functions causes unrealistically higher resource requirements for the growth of biomass. On the other hand, the use of SU causes comparatively lower resource requirements for the growth of biomass. In addition, with the
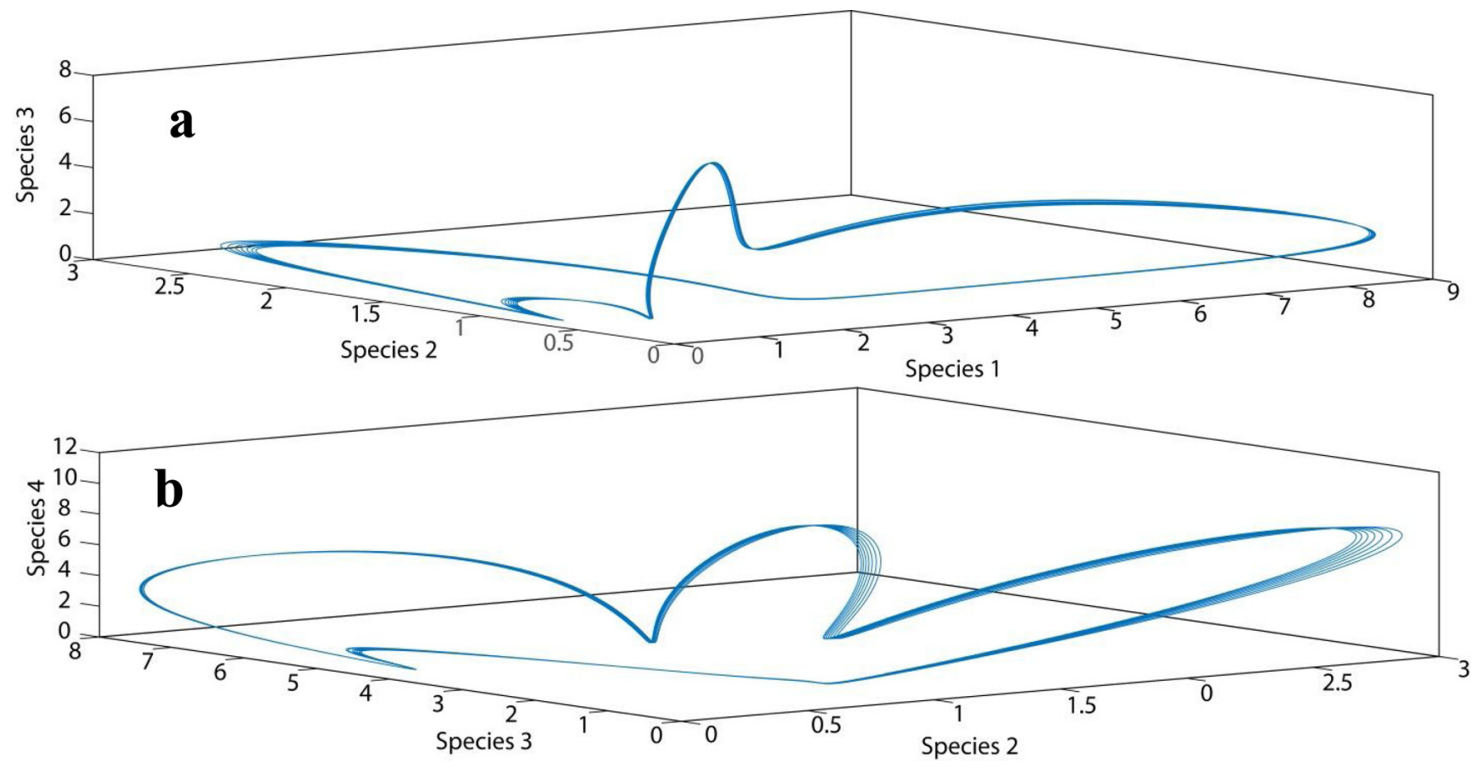

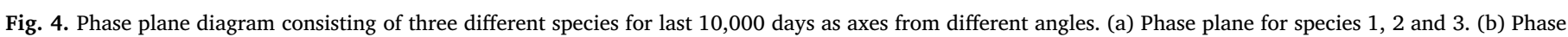
plane for species 2, 3 and 4 . 

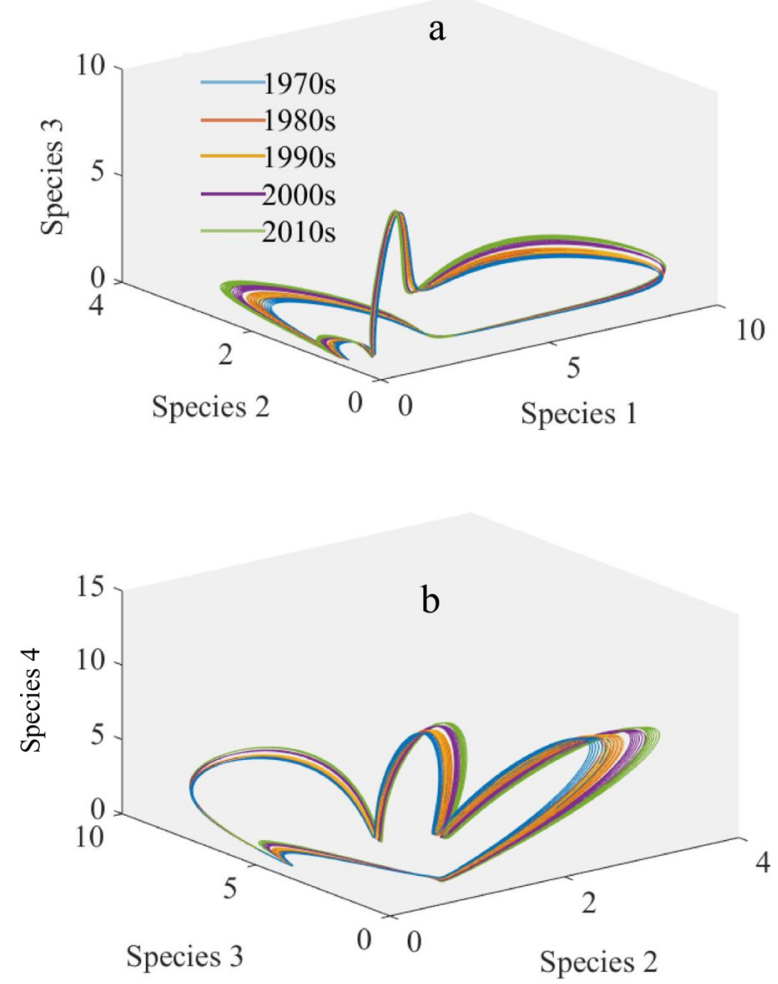

Fig. 5. Phase plane diagram consisting of three different species for last 10,000 days as axes from different angles. (a) Phase plane for species 1, 2 and 3. (b) Phase plane for species 2, 3 and 4. Different colors in both phase plane diagram indicate the trajectories of species generated by the model with different environmental forcing.

SU based approach the production of biomass changes smoothly. Secondly, we found that our model based on the SU formulation exhibits competitive exclusion and stable coexistence of species in an equilibrium or in periodic as well as chaotic oscillations. Thirdly, we found that the parameters of our model with SU are relatively less sensitive then the model with product of several Monod functions (Fig. S2). Very small variations in the parameters (i.e. $3-4 \%$ of any parameters of the model) in the model with product of several Monod functions change the qualitative nature of the results (i.e. breaks the supersaturation state). However, in our model with the SU based approach, comparatively wide variations in the parameters values (i.e. change in at least $20 \%$ of any parameter of the model) do not break the supersaturation state. Hence, the dynamics of the SU based model is more robust with respect to changes in the parameters. With these findings i.e. lower resource requirements for the production of biomass, ability to produce all possible outcomes of a competition model and comparatively less sensitivity to variation in parameter values we can conclude that our model performs better than the model with product of several Monod functions.

\section{Discussion}

We used and extended the multiple species-multiple resources competition model developed by Dutta et al. (2014) employing an SUbased growth rate to simulate phytoplankton species dynamics in a multiple resource limited system. Based on the Helgoland Roads data we were able to find for the first time a realistic parameterization of the model for competition of the plankton species where species growth is formulated based on SU. The primary goal of this study was to test whether the number of coexisting species can exceed the number of known limiting resources in a multiple resource-limited planktonic ecosystem. We considered a system where four species compete for three interactive essential resources in order to examine this hypothesis.

Nutrient limitation of oceanic primary production exerts a fundamental control on marine food webs and the flux of carbon in the ocean (Moore et al., 2013). Phytoplankton growth and stoichiometry depend on the availability of multiple nutrients (Klausmeier et al., 2004). The historical, conceptual understanding of nutrient limitation is essentially one of single-nutrient limitation that is derived from Liebig's Law of the Minimum (de Baar, 1994). Liebig's Law of the Minimum was developed to describe the constraints on the production of individual crop plants, but not the biologically diverse communities of plants and ecosystems to which Liebig's Law has been extended (Danger et al., 2008). However, research finds that the growth of phytoplankton of the ocean is not limited by a single nutrient, but by multiple nutrients simultaneously (Browning et al., 2017). The multiple limitation assumes interactive-essential resources, i.e. it considers interactions between

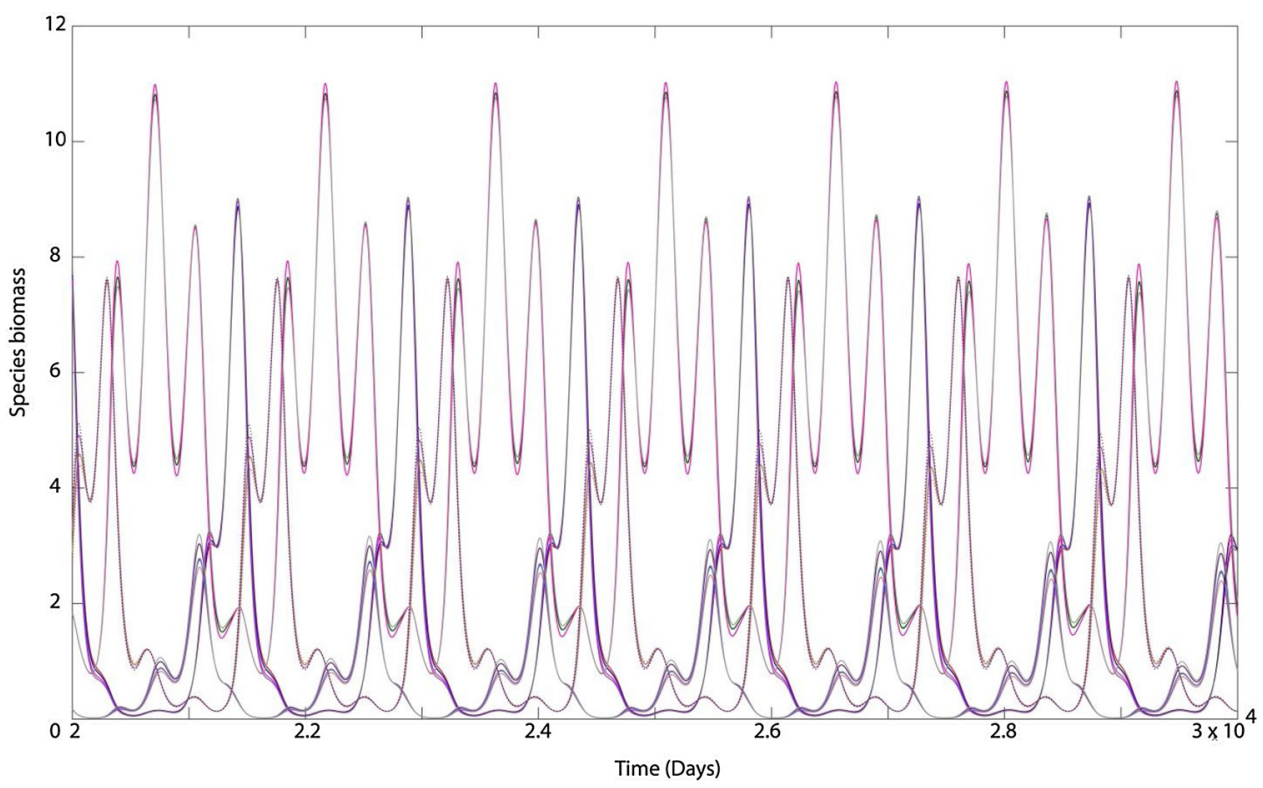

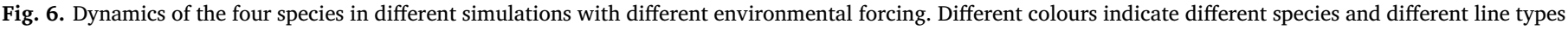
indicate different environmental forcing. 


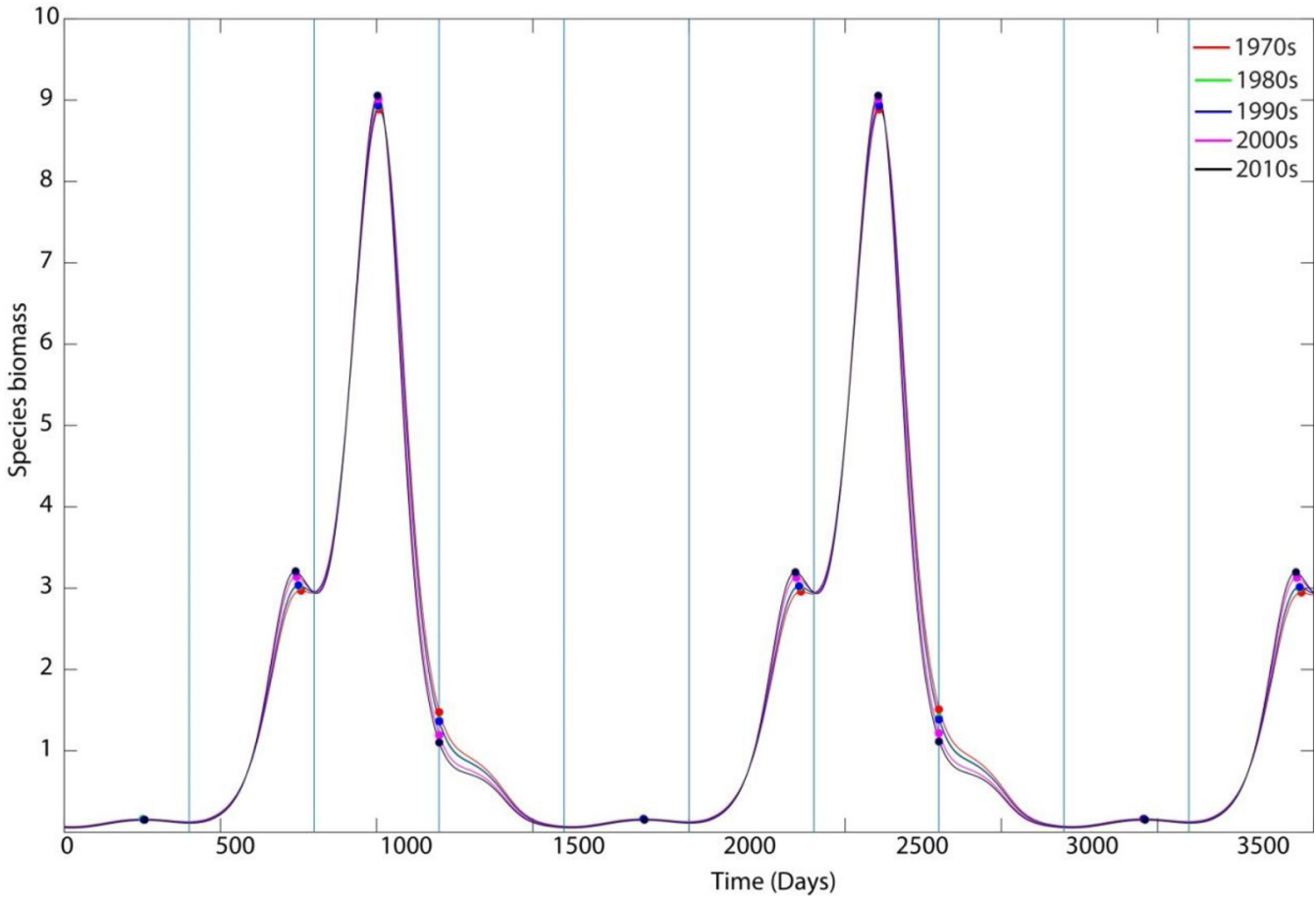

Fig. 7. Change in dominance and turnover time of species 1 in different simulations for last 10 years of simulations for the same species. Vertical blue lines indicate the one year interval. Different dots indicate the peak at every 365 days (For interpretation of the references to colour in this figure legend, the reader is referred to the web version of this article). limiting nutrients and predicts smooth transitions from a limitation by one nutrient to a limitation by another (Sperfeld et al., 2012). According to the multiple resource limitation hypothesis, all nutrients limit growth to some extent but the strength of a limitation by a particular nutrient depends on the supply relative to the demand (Sperfeld et al., 2012). Nitrogen, phosphorus, silicon, inorganic carbon, iron, and light are examples of abiotic essential resources which can limit the growth of phytoplankton. According to the multiple resource limitation hypothesis phytoplankton growth should be constrained by more than one resource simultaneously and is subject to interactive effects between co-limiting resources. At co-limiting conditions, growth rates predicted by the co-limitation hypothesis are lower than those predicted by applying Liebig's law, i.e. by assuming strictly essential resources (Sperfeld et al., 2012). Therefore, in our study we considered simultaneous limitation of resources for phytoplankton.

The competition model for phytoplankton we studied in this paper is based on a SU growth rate to take the interactive effects of multiple nutrients (i.e. $\mathrm{SiO}_{2}, \mathrm{NO}_{3}$ and $\mathrm{PO}_{4}$ ) into account. By employing the $\mathrm{SU}$ concept to a phytoplankton community, we pursue a much more mechanistic approach than the product of Monod or von Bertalanffy functions which is often used in other competition models to account for co-limitation (Huisman and Weissing 2002). A SU can bind an arbitrarily large number of nutrients to the phytoplankton body and transfer them into phytoplankton biomass. Phytoplankton uptake the nutrient which arrives first and subsequently, uptake other nutrients in next steps. The phytoplankton biomass can only grow when phytoplankton uptake all nutrients. Most of the earlier studies on multiple resource competition have considered Liebig's minimum law to model the phytoplankton growth kinetics. Previously, the product law growth rate, which is determined by the product of, e.g., several Monod terms, has been used for that purpose (Huisman and Weissing, 2002). But this product law growth rate has a serious methodological disadvantage as it requires that the concentrations of all resources must be unrealistically high. This results in a lack of a good fit to experimental data (Droop, 2009; Kooijman, 1998; Sperfeld et al., 2012). Recent experimental results on resource co-limitation theory applied to herbivorous consumers have shown that species growth kinetics based on the concept of SU fits the data better than the product law growth rate (Sperfeld et al., 2012). Thus we formulated species growth based on SU to take into account the multiple resource limitation rather than using the product of several Monod functions.

In our study we found lower resource requirements for the production of biomass, the ability to produce all possible outcomes of a competition model and comparatively less sensitivity to variation in parameter values. Hence, we can conclude that our model performs better than the model with product of several Monod functions. This suggests that SU is a much more suitable approach to model the colimitation by several resources.

Our study shows that the number of plankton species coexisting can indeed exceed the number of limiting resources in a multiple resourcelimited system when species growth is formulated based on SU concept. The periodic biomass oscillations resulting from the competition of four species for three resources allow the coexistence of more species than the number of resources in the long-term. Huisman and Weissing (1999), Huisman et al. (2001), Huisman and Weissing (2002) and Baer et al. (2006) found that competition for resources produces periodic as well as chaotic fluctuations in biomass which enable species to coexist. In contrast to their studies, we considered a planktonic ecosystem in seasonally changing environment and showed this periodic forcing does not prevent supersaturation to occur. The periodic oscillation in species biomass which is observed in nature due to the seasonal forcing and also found in this study is a plausible mechanism for species coexistence. Our finding is compatible with the findings of Dutta et al. (2014) who also found that more species than limiting resources may coexist by producing a periodic oscillation in their biomass but without seasonal forcing. In addition, variation in resource requirements of species causes the variation in interspecific competition and leads to niche differentiation constituting another cause for stable coexistence (Büchi and Vuilleumier, 2014). In our model, species exhibit non-linearity in their functional responses. This non-linearity reduces competition among the species for resources and allows stable coexistence (Armstrong and McGehee, 1980).

We also observed that the long-term species coexistence holds, even if we change the forcing within a realistic range. Dutta et al. (2014), Huisman and Weissing (1999), Huisman et al. (2001), Feng et al. (2011), Schippers et al. (2001) and Kishi and Nakazawa (2013) also changed the range of environmental forcing with respect to nutrients in the model and found a supersaturated state in species coexistence 
within a certain range. Environmental forcing exceeding a certain range limited the more species coexistence than the number of limiting resources in their studies.

Comparison of model outputs with empirical data: Our findings show that in the supersaturation state the identity of the dominant species within the community varies overtime. This is in line with the findings of Dutta et al. (2014) and Huisman and Weissing (1999). Moreover, we observed cycles in species biomass with peaks every couple of years and changes in the identity of the dominant species. In order to check these model implications, we analyzed the long-term real phytoplankton data sets. To infer a relation between model outputs and empirical data we should simply find similar patterns of peaks in species abundance and repetition of peaks in empirical data as it was found in our model simulations. To do this, firstly, we plotted the long-term changes in species abundance to check their behaviour i.e. changes in species dominance and the timing of peak abundances. Secondly, we observed a change in species abundance at different environmental conditions. The long-term phytoplankton data (Fig. 1) from Helgoland Roads shows that Asterionellopsis glacialis, Thalassionema nitzschioides, Brockmanniella brockmannii, Odontella aurita and $O$. regia species show major peaks at several years interval which is similar to our model simulations. A. glacialis shows peak in abundance sometimes after 2 to 3 years interval and sometimes after 5 to 8 years interval. T. nitzschioides generally peaks after 2 to 4 years interval but sometimes 7 to 8 years interval and $B$. brockmannii generally shows the peak in abundance every 4 to 6 years interval. The diatoms Rhizosolenia imbricate and Guinardia delicatula show the peak in their abundance during the period from June to August and therefore, are known as summer species. On the other hand, Paralia sulcuta shows the peak in abundance during winter. These species show a major peak in abundance at least once in a year. In the Helgoland Roads data sets there are many other species with similar behaviour which are in accordance with our model implications.

However, in order to check the quantitative nature of comparisons between model outputs and the observed phytoplankton data from the Helgoland Roads, we used the Kappa statistics (McHugh, 2012). The kappa statistics is frequently used to test interrater reliability and it represents the extent to which the data collected in the study are correct representations of the variables measured (McHugh, 2012). It is generally thought to be a more robust measure than simple percent agreement calculation, as kappa statistics takes into account the possibility of the agreement occurring by chance. The kappa values range from -1 to +1 and Cohen's suggested that a score as low as 0.41 might be acceptable (Marston, 2009). Here we used the kappa statistics to compare the signals of phytoplankton dynamics (i.e. identity of dominant species and repetition of peak in biomass) produced by the model with the signals in the observed data from the Helgoland Roads. The method proceeds as follows: firstly, we identify and extract phytoplankton events (i.e. identity of dominant species and repetition of peak in biomass) from the time series data and model outputs. As a next step, we applied the kappa statistics to the events extracted from the observed data and model simulations to see the percentage of agreements between these two data sets. The kappa values for the events identity of dominant species and repetition of peak were found 0.86 and 0.84 , respectively. These suggest the excellent agreements between the signals produced by the model and signals found in the observed data.

Similar type of repetition of peak in biomass and dominance of species also observed by Dakos et al. (2009) where this type of scenarios were shown due to chaos within the community. Dakos et al. (2009) observed peak abundance of Asterionella kariana varies year to year and G. delicatula can display several peaks per year at the time series of the Dutch coastal zone. A diatom species, A. formosa in Lake Windermere of UK also showed year to year variation in the timing of dominance (Maberly et al., 1994). Another study (Smayda, 1998) on time series data of phytoplankton in Narragansett Bay of USA confirms the irregular timing of the peak in abundance of species (i.e. A. glacialis) and a regular peak at certain time interval (i.e. Thalassiosira nordenskioeldii peak at 5-year interval).

These types of scenarios of peaks in species biomass might evolve due to the timing and arrival flux of different resources, and speciesspecific requirements for those resources. Arrival flux of a particular resource might cause the peak of a species that has the highest requirement for that resource. For example, Freund et al. (2006) found that resetting the temperature within the seasonal cycle causes an interchange of bloom and non-bloom modes of phytoplankton which might hold true for nutrients also.

We examined the response of phytoplankton species in different environmental conditions using the Helgoland Roads Time Series data sets. We present results for four taxa: Chaetoceros spp, A. glacialis, P.sulcata and T. nitzschioides (Fig. S3). All these species change their abundance in different environmental conditions. For example, C. spp shows an increase in abundance in recent decades while T. nitzschioides shows a decrease. In our model simulations though we found that species change their biomass in different environmental forcing, however, these changes are not significant. We note that the significance of our claimed signature on species peak abundance, repetition of peak and response of species at different environmental conditions is mostly qualitative in nature. A more quantitative study would require a more careful statistical evaluation between model outputs and observed data, but this is beyond the scope of this investigation. However, we consider the analyses described in this paper to be a first valuable step towards a better understanding of species coexistence integrating model simulations and empirical data.

\section{Conclusion}

In conclusion, what have we learned? More phytoplankton species can coexist than the number of limiting resources even when the system is simultaneously co-limited by resources and species growth is formulated based on SU. This supersaturated state can evolved due to periodic fluctuation in species biomass, variation in interspecific competition and niche configuration, nonlinear functional response of species and resource supply rate. This coexistence of more species than limiting resources occurs considering additionally seasonal variation of environmental condition.

Our study is the extension of the species competition model developed by Dutta et al. (2014). In this study we considered phytoplankton species in a shallow sea ecosystem, included seasonal temperature and light forcing and, most importantly, we considered a new real parameter set for the model. In addition, we compared our model outputs with observed data form the Helgoland Roads data sets. This is the first explanation for phytoplankton species coexistence in a shallow sea system which violates the competitive exclusion principle in a multiple resource-limited system. This study has created new avenues for further research. For example, species coexistence study taking consideration of two diatom species competing for silicate, nitrate and phosphate, and two dinoflagellate species competing for nitrate and phosphate would be interesting. Addition of stochastic terms, and combination generalist and specialist species in the model will be our next goal. Addition of more species with fast and slow growth rates at the beginning and also at different time intervals of the simulations might produce more exciting insights on phytoplankton species coexistence.

\section{Acknowledgments}

We acknowledge the present and past crews of the research vessels 'Aade' and 'Ellenbogen' for their unfailing provision of samples. We also 
thank Silvia Peters, Kristine Carstens and all colleagues of time series group for analyzing phytoplankton samples and nutrients. S. Sarker thanks Ramesh Arumugam for his help with the simulations. C. L. Meunier was financed by the Bundesministerium für Bildung und Forschung (BMBF grant no. 01LN1702A).

\section{Appendix A. Supplementary data}

Supplementary material related to this article can be found, in the online version, at doi:https://doi.org/10.1016/j.ecolmodel.2018.05. 021.

\section{References}

Armstrong, R.A., McGehee, R., 1980. Competitive exclusion. Am. Nat. 115, 151-170. Baer, M.S., Li, B., Smith, L.H., 2006. Multiple limit cycles in the standard model of three species competition for three essential resources. J. Math. Biol. 52, 745-760.

Browning, T.J., Achterberg, E.P., Rapp, I., Engel, A., Bertrand, E.M., Tagliabue, A., Moore, C.M., 2017. Nutrient co-limitation at the boundary of an oceanic gyre. Nature 551, 242.

Büchi, L., Vuilleumier, S., 2014. Coexistence of specialist and generalist species is shaped by dispersal and environmental factors. Am. Nat. 183, 612-624.

Dakos, V., Benincà, E., van Nes, E.H., Philippart, C.J.M., Scheffer, M., Huisman, J., 2009. Interannual variability in species composition explained as seasonally entrained chaos. Proc. R. Soc. Lond. B: Biol. Sci. 276, 2871-2880.

Danger, M., Daufresne, T., Lucas, F., Pissard, S., Lacroix, G., 2008. Does Liebig's law of the minimum scale up from species to communities? Oikos 117, 1741-1751.

de Baar, H.J.W., 1994. von Liebig's law of the minimum and plankton ecology (1899-1991). Prog. Oceanogr. 33, 347-386.

Droop, M.R., 2009. The nutrient status of algal cells in continuous culture. J. Mar. Biol. Assoc. U. K. 54, 825-855.

Dutta, P.S., Kooi, B.W., Feudel, U., 2014. Multiple resource limitation: nonequilibrium coexistence of species in a competition model using a synthesizing unit. Theor. Ecol. 7, 407-421.

French, R.H., Cooper, J.J., Vigg, S., 1982. Secchi Disc Relationships. Water Resour. Bull. $18,121-123$.

Feng, Z., Liu, R., Qiu, Z., Rivera, J., Yakubu, A.-A., 2011. Coexistence of competitors in deterministic and stochastic patchy environments. J. Biol. Dynamics 5, 454-473.

Freund, J.A., Mieruch, S., Scholze, B., Wiltshire, K., Feudel, U., 2006. Bloom dynamics in a seasonally forced phytoplankton-zooplankton model: trigger mechanisms and timing effects. Ecol. Complex 3, 129-139.

Gilks, W.R., Richardson, S., Spiegelhalter, D., 1995. Markov Chain Monte Carlo in Practice. Taylor \& Francis, Chapman \& Hall/CRC, London, UK.

Gleeson, S.K., Tilman, D., 1992. Plant allocation and the multiple limitation hypothesis. Am. Nat. 139, 1322-1343.

Grasshoff, K., Almgreen, T., 1976. Methods of Seawater Analysis. V C H Publishers, Incorporated.

Hardin, G., 1960. The competitive exclusion principle. Science 131, 1292-1297.

Hartig, F., Munkemuller, T., Johst, K., Dieckmann, U., 2014. On the sympatric evolution and evolutionary stability of coexistence by relative nonlinearity of competition. PloS One 9, e94454.

Hubbell, S.P., 2001. The Unified Neutral Theory of Biodiversity and Biogeography (MPB32). Princeton University Press.

Huisman, J., Johansson, A.M., Folmer, E.O., Weissing, F.J., 2001. Towards a solution of the plankton paradox: the importance of physiology and life history. Ecol. Lett. 4, 408-411.

Huisman, J., Weissing, F.J., 1999. Biodiversity of plankton by species oscillations and chaos. Nature 402, 407-410.

Huisman, J., Weissing, F.J., 2002. Oscillations and chaos generated by competition for interactively essential resources. Ecol. Res. 17, 175-181.

Hutchinson, G.E., 1961. The paradox of the plankton. Am. Nat. 95, 137-145.

Kishi, S., Nakazawa, T., 2013. Analysis of species coexistence co-mediated by resource competition and reproductive interference. Popul. Ecol. 55, 305-313.

Klausmeier, C.A., Litchman, E., Levin, S.A., 2004. Phytoplankton growth and stoichiometry under multiple nutrient limitation. Limnol. Oceanogr. 49, 1463-1470.

Kooi, B.W., Kuijper, L.D.J., Kooijman, S.A.L.M., 2004. Consequences of symbiosis for food web dynamics. J. Math. Biol. 49, 227-271.

Kooijman, S.A.L.M., 1998. The synthesizing unit as model for the stoichiometric fusion and branching of metabolic fluxes. Biophys. Chem. 73, 179-188.

Kooijman, S.A.L.M., 2010. Dynamic Energy Budget Theory for Metabolic Organisation. Cambridge University Press.

Laird, R.A., Schamp, B.S., 2006. Competitive intransitivity promotes species coexistence. Am. Nat/ 168, 182-193.

Li, Q.P., Franks, P.J.S., Landry, M.R., Goericke, R., Taylor, A.G., 2010. Modeling phytoplankton growth rates and chlorophyll to carbon ratios in California coastal and pelagic ecosystems. J. Geophys. Res. 115 n/a-n/a.

Liebig, J., 1840. Organic Chemistry in Its Applications to Agriculture and Physiology, Taylor and Walton.

Maberly, S.C., Hurley, M.A., Butterwick, C., Corry, J.E., Heaney, S.I., Irish, A.E., Jaworski, G.H.M., Lund, J.W.G., Reynolds, C.S., Roscoe, J.V., 1994. The rise and fall of Asterionella formosa in the South Basin of Windermere: analysis of a 45-year series of data. Freshw. Biol. 31, 19-34.

Marston, L., 2009. Introductory Statistics for Health and Nursing Using SPSS. SAGE Publications.

McHugh, M.L., 2012. Interrater reliability: the kappa statistic. Biochemia Medica 22, $276-282$.

Moore, C.M., Mills, M.M., Arrigo, K.R., Berman-Frank, I., Bopp, L., Boyd, P.W., Galbraith, E.D., Geider, R.J., Guieu, C., Jaccard, S.L., Jickells, T.D., La Roche, J., Lenton, T.M., Mahowald, N.M., Maranon, E., Marinov, I., Moore, J.K., Nakatsuka, T., Oschlies, A., Saito, M.A., Thingstad, T.F., Tsuda, A., Ulloa, O., 2013. Processes and patterns of oceanic nutrient limitation. Nat. Geosci. 6, 701-710.

Muller, E.B., Nisbet, R.M., Kooijman, S.A.L.M., Elser, J.J., McCauley, E., 2001. Stoichiometric food quality and herbivore dynamics. Ecol. Lett. 4, 519-529.

Poggiale, J.C., Baklouti, M., Queguiner, B., Kooijman, S.A.L.M., 2010. How far details are important in ecosystem modelling: the case of multi-limiting nutrients in phytoplankton-zooplankton interactions. Phil. Trans. R. Soc. B: Biol. Sci. 365, 3495-3507.

R Development Core Team, 2010. R: A Language and Environment for Statistical Computing. R Foundation for Statistical Computing, Vienna.

Raabe, T., Wiltshire, K.H., 2009. Quality control and analyses of the long-term nutrient data from Helgoland Roads, North Sea. J. Sea Res. 61, 3-16.

Roelke, D., Augustine, S., Buyukates, Y., 2003. Fundamental predictability in multispecies competition: the influence of large disturbance. Am. Nat. 162, 615-623.

Roelke, D.L., Eldridge, P.M., 2008. Mixing of supersaturated assemblages and the precipitous loss of species. Am. Nat. 171, 162-175.

Sakavara, A., Tsirtsis, G., Roelke, D.L., Mancy, R., Spatharis, S., 2017. Lumpy species coexistence arises robustly in fluctuating resource environments. Proc. Natl. Acad. Sci. http://dx.doi.org/10.1073/pnas.1705944115. USA.

Scheffer, M., van Nes, E.H., 2006. Self-organized similarity, the evolutionary emergence of groups of similar species. Proc. Natl. Acad. Sci. 103, 6230-6235.

Schippers, P., Verschoor, A.M., Vos, M., Mooij, W.M., 2001. Does "supersaturated coexistence" resolve the "paradox of the plankton"? Ecol. Lett. 4, 404-407.

Segura, A.M., Calliari, D., Kruk, C., Conde, D., Bonilla, S., Fort, H., 2011. Emergent neutrality drives phytoplankton species coexistence. Proc. Biol. Sci. R. Soc. 278, 2355-2361.

Smayda, T.J., 1998. Patterns of variability characterizing marine phytoplankton, with examples from Narragansett Bay. ICES J. Mar. Sci. 55, 562-573.

Sperfeld, E., Martin-Creuzburg, D., Wacker, A., 2012. Multiple resource limitation theory applied to herbivorous consumers: Liebig's minimum rule vs. interactive co-limitation. Ecol. Lett. 15, 142-150.

Sturtz, S., Ligges, U., Gelman, A., 2005. R2WinBUGS: a package for running WinBUGS from R. J. Stat. Softw. 12, 1-16.

Thomas, A., O'Hara, B., Ligges, U., Sturtz, S., 2006. Making BUGS open. R News 6, 12-17. Vandermeer, J.H., 1972. Niche theory. Annu. Rev. Ecol. Syst. 3, 107-132.

Wiltshire, K.H., Dürselen, C.-D., 2004. Revision and quality analyses of the Helgoland Reede long-term phytoplankton data archive. Helgoland Mar. Res. 58, 252-268.

Wiltshire, K.H., Kraberg, A., Bartsch, I., Boersma, M., Franke, H.D., Freund, J., Gebuhr, C., Gerdts, G., Stockmann, K., Wichels, A., 2010. Helgoland Roads, North Sea: 45 years of change. Estuaries Coasts 33, 295-310. 